\title{
Real-time PCR quantification of human telomerase reverse transcriptase (hTERT) in colorectal cancer
}

Running title: telomerase in colorectal cancer

Said Saleh, BSc, $\mathrm{PhD}^{1}$

Alfred King-Yin Lam, MBBS, PhD, MD, FRCPA ${ }^{2}$

Yik-Hong Ho, MBBS, MD, FRACS ${ }^{1}$

${ }^{1}$ Department of Surgery (School of Medicine) and North Queensland Centre for Cancer

Research (Australian Institute of Tropical Medicine), James Cook University, Townsville,

Queensland 4811, Australia

${ }^{2}$ Discipline of Pathology, Griffith Medical School, Medicine and Oral Health Centre, Gold

Coast, Queensland 4215, Australia

Correspondence and/or reprint requests:

Professor Alfred Lam

Griffith Medical School

Medicine and Oral Health Centre

PMB 50 GCMC Bundall

Gold Coast QLD 9726

Australia

Phone +61 756780718 Fax +61756780708

E-mail: a.lam@griffith.edu.au

\section{Acknowledgement of grant support}

The project was supported by a grant from the Queensland Cancer Fund.

The first two authors contributed equally to the work 


\begin{abstract}
Ains: Human telomerase reverse transcriptase (hTERT) is believed to a reliable marker for telomerase activity. The expression of telomerase activity has not been investigated in a consecutive series of patients with colorectal adenocarcinoma in North Queensland. The objective of this study was to evaluate the significance of hTERT mRNA expression in colorectal adenocarcinoma in North Queensland.
\end{abstract}

Methods: Matched samples of tumour and adjacent nontumourous mucosa samples from 53 colorectal carcinomas and 9 colorectal adenomas were collected. In all these samples, RNA was extracted and then transcribed to cDNA. Real-time polymerase chain reaction (RT-PCR) was used to quantitate expression level of hTERT mRNA. The findings were correlated with the clinicopathological features of patients with these tumours prospectively collected into a computerized database.

Results: hTERT mRNA was expressed in all tumour samples. The level of expression in the colorectal adenocarcinomas was significantly higher than the corresponding nontumorous mucosa ( $p=0.009$, t-test). The level of expression in the adenocarcinomas was slightly higher than those of adenomas but the difference was not statistically significant. Higher level of hTERT expression was often noted in the adenocarcinomas arising from left colon \& rectum when compared with those from the right colon $(\mathrm{p}=0.029)$

Conclusions: Colorectal adenocarcinoma revealed expression of telomerase hTERT mRNA, which was detected quantitatively by real-time PCR. hTERT could be a potential biomarker for colorectal cancer. The difference between proximal and distal colorectum in hTERT expression could account for their known different clinical behaviour. 


\section{Introduction}

Proliferation of the cells is carefully regulated by many factors such as gene controlled cell division, repair, and apoptosis. Break down in one of the regulation controls occasionally happens due to viral infection, DNA methylation, and sequence alteration. This leads to unregulated cell division fashion and ultimately forms a tumour. Colorectal cancer is one of the common diseases, which is developed in a multi-step process and associated with genetic alterations $(1,2)$. One of the characteristic features of cancer is telomerase activation.

Human telomerase is a ribonucloprotein composed of two components, human telomerase RNA (hTR) and human telomerase reverse transcriptase (hTERT). Both components are essential for telomerase activity. This unique enzyme uses its RNA as a template to add telomeric repeats at the end of chromosome ends in order to compensate for telomere loss during cell division $(3,4)$. The cell is able to continue proliferation because telomerase stabilizes the chromosome ends from degradation and fusion. It is believed that telomerase reactivation in the cell is associated with carcinogenesis and is a critical step in the tumour immortalization process (5-7).

Telomerase activity has been found in more than $85 \%$ of all human tumours, as well as germ and stem cells. In normal cells, it is either low or undetectable level. A polymerase chain reaction (PCR)-based method, telomeric repeat amplification protocol (TRAP) assay, has been developed to detect telomerase activity $(8,9)$. hTERT expression is restricted to cancer cells and closely associated to telomerase activity. This indicates that hTERT expression is the ratelimiting determinant of telomerase and could be used as means of measuring telomerase activity (10-12). In colorectal cancer, conflicting results have been reported for telomerase activation and its correlation with clinical parameters. Despite absence of telomerase in normal mucosa, these studies have demonstrated the activation of telomerase occurs in adenomas and telomerase 
level is further increased in carcinomas $(13,14)$. Other reports revealed a detectable level of telomerase activity in normal mucosa (15-17). A few studies have shown a significant association between telomerase and clinicopathological features during cancer progression (1618), whereas others failed to find such a relationship $(13,15)$.

Although telomerase activity has been detected in colorectal cancer, much remains unknown about hTERT expression and correlation to tumour progression and clincopathological features. In this study, we evaluated expression level of hTERT mRNA in tumour tissues and corresponding adjacent non-tumour mucosa from patients with colorectal adenocarcinomas and adenomas by using real-time reverse transcriptase polymerase chain reaction. We also analyzed the relationship between hTERT expression level and clinicopathological features of the patients. 


\section{Materials and Methods}

\section{$\underline{\text { Patient selection and sample collection }}$}

Patients with histologically confirmed colon and rectal tumours were prospectively and consecutively recruited into the study. Consent was given according to the hospital human ethics committee approval. The clinical diagnostic, staging, surgical and pathological data were collected in a standardized manner, using a computerized database. Management was by a preagreed standardized multidisciplinary protocol supervised by a senior specialist colorectal surgeon. None of the rectal cancer patients had undergone neoadjuvant radiotherapy or chemotherapy prior to surgery. Pathological diagnosis was also standardized and reviewed by committee criteria, chaired by a senior academic pathologist with a special interest in the field. At the time of surgery, the tissue from the tumour and normal colorectal mucosa from the proximal resection margin were promptly sampled from the resected specimen. These samples were preserved in RNAlater (Ambion, Austin, TX, USA) within 10 minutes of surgery to prevent degradation of the RNA. They were stored at $-80^{\circ} \mathrm{C}$ for the experiment.

\section{RNA extraction}

Total RNA was extracted from the 10-25 mg of tested tissue by disruption and homogenization using High Pure RNA Tissue Kit (Roche Diagnostics, Mannheim, Germany). DNase I was used to digest DNA contamination. Quality of the RNA was checked by agarose gel electrophoresis to rule out degraded RNA.

\section{cDNA preparation}

Purified total RNA was reverse transcribed in a total volume of $25 \mu \mathrm{l}$ using random primer at a ratio 2 to 1 RNA and transcriptor reverse transcriptase (Roche Diagnostics, Mannheim, Germany). The reaction mixture was incubated for $10 \mathrm{~min}$ at $25{ }^{0} \mathrm{C}$ first, $60 \mathrm{~min}$ at 
$50{ }^{\circ} \mathrm{C}$, and followed by enzyme inactivation step for $5 \mathrm{~min}$ at $85^{\circ} \mathrm{C}$. The cDNA samples were stored at $-20{ }^{0} \mathrm{C}$ until use. 
$\underline{\text { Real time quantification PCR }}$

Rotor gene system (Corbett Research, Sydney, Australia) was used to run real-time quantification polymerase chain reaction. Absolute quantification assay was chosen to analyse both hTERT (target) and glyceraldehydes 3-phosphate dehydrogenase (GAPDH, endogenous control) genes expression. PCR was performed in a total volume of $20 \mu \mathrm{l}$ reaction mixture containing 1x TaqMan universal master mix with AmpErase uracil N-glycosylase (Applied Biosystems, Foster City, USA)(to prevent the re-amplification of carry-over PCR products), $600 \mathrm{nM}$ of each primer, 200nM TaqMan probe, and $1 \mu \mathrm{l}$ of unknown cDNA or $2 \mu \mathrm{l}$ of standard template. All the samples (unknown or standard) were run in duplicate and accompany with non- template control. Thermal cycling conditions included $2 \mathrm{~min}$ at $50{ }^{\circ} \mathrm{C}$ and $10 \mathrm{~min}$ at $95{ }^{0} \mathrm{C}$, followed by 45 cycles at $95{ }^{0} \mathrm{C}$ for $15 \mathrm{sec}$ and $60{ }^{0} \mathrm{C}$ for $60 \mathrm{~min}$.

Primers and probes

The primer set and probe for amplification of hTERT (GenBank accession number AF015950) and GAPDH, (GenBank accession number NM_002046) genes were designed using GenScript web site/design tool (www.genscript.com/ssl-bin/app/primer) (Table 1). The primers for both genes, placed in different exons, were checked by conventional PCR to ensure they did not amplify genomic DNA. The probes contained 6-carboxyflourscein (FAM) as fluorescent reporter dye, and 6-carboxytetramethyl-rhodamine (TAMRA) as quencher for its light emission spectrum. These probes were purchased from Sigma Genosys (Wollands, TX, USA). During the extension phase of PCR, the probe hybridized to the target sequence and was then cleaved due to the 5' to 3' exonuclease activity of Taq polymerase. The increase in fluorescence signal of the reporter was proportional to the amount of specific PCR products, providing highly accurate and reproducible quantification. 


\section{$\underline{\text { Standard curve }}$}

For absolute quantitation assay, a standard curve was constructed from known concentration of DNA sample. A segment of $290 \mathrm{bp}$ hTERT gene was generated by conventional RT-PCR. The PCR-product was checked on agarose gel for unspecific amplification, purified by High Pure PCR Product Kit (Roche Diagnostics, Mannheim, Germany), and then accurately quantified by ultraviolet-spectrophotometer. The concentration of known DNA sample was converted to molecules per microliter. This known standard sample was adjusted to $0.6 \times 10^{10}$ molecule/ $\mu \mathrm{l}$ and then serially diluted by $1 / 10$ down to $0.6 \times 10$. Two $\mu \mathrm{l}$ of each dilution $\left(10^{6}\right.$ to $\left.10^{1}\right)$ in duplicate was used as a template for real time PCR.

For each sample, the expression level of hTERT and GAPDH were quantified as a copy numbers (per reaction) using the standard curve (Figure 1). Expression level of hTERT for each sample was normalized by dividing the copy number of hTERT by the corresponding copy number of GAPDH. (Normalized hTERT = copy number of hTERT $_{\text {sample }}$, copy number of GAPDH sample $_{\text {x100) }}$

\section{$\underline{\text { Statistical Analysis }}$}

Expression difference of hTERT mRNA between tumour and matched non-tumour samples were done using t- test. Correlation between expression level of hTERT and clinicopathological features of the patients was analysed by Kruskall-Wallis and Mann-Whitney tests. Fisher's exact test was used to assess expression level in proximal and distal tumours. The significant level was taken at $\mathrm{p} \leq 0.05$. All statistical tests were performed with the program, Statistical Package for Social Sciences (SPSS version 12.0, Chicago, IL). 


\section{Results}

Between January 2003 to December 2005, 53 patients (32 men, 21 women) with colorectal adenocarcinomas with a median age of 68 (range 37-94) were recruited. The salient clinical and histological features of patients with colorectal adenocarcinoma are shown (Table 2). The 9 patients (3 men, 6 women) with colorectal adenomas had a median age of 66 .

hTERT mRNA was expressed in all tumour samples. The level of expression in tumour tissues was statistically significant $(\mathrm{P}=0.009$, t-test $)$ in comparison with matched adjacent mucosa in the same patient. The range of hTERT mRNA expression level was 0.111-3.033 (median 0.685, mean 0.992) in colorectal adenocarcinoma and 0-1.584 (median 0.615, mean 0.671) in adjacent mucosa samples (Figure 2). Of the 53 colorectal tumour samples, 30 (57\%) were found to express higher hTERT mRNA, while 23 (43\%) expressed less hTERT mRNA compared with matched adjacent mucosa. In patients with colorectal adenomas $(n=9)$, the expression level of hTERT was not significantly higher than the corresponding nontumorous mucosa. The range of hTERT mRNA expression level was 0.011-1.977 (median 0.474). hTERT expression in the adenocarcinomas was slightly higher than those of the adenomas but the difference was not statistically significant. In nontumorous mucosa, expression level of hTERT decreased with age (data not shown). There was no significant association between colorectal adenocarcinoma hTERT mRNA expression level and patients’ age, gender, tumours' differentiation, size, site and stage (Table 2). The mean value of hTERT copy number for colorectal adenocarcinoma samples was 0.992 . In order to study the possible correlation between hTERT expression and the location of colorectal tumours, a cut-off value of 1 was considered to categorize hTERT as either low or high. Of the 53 tumour samples, $40 \%$ expressed high level and 60\% low level of hTERT mRNA. 36\% of patients with left colon \& rectum (descending colon, sigmoid colon and rectum) expressed high level of hTERT compared 
with 4\% patients with right colon cancers (caecum, ascending colon and transverse colon)

(Table 3). Thus, significant difference was found between hTERT expression and the location of the tumour $(\mathrm{P}=0.029)$. 


\section{Discussion}

Telomerase activity is strongly linked with the development of tumour and may play a critical role as a biomarker for early detection of cancer. TRAP assay has been used to detect telomerase activity. Recently, real time PCR has been used to detect and evaluate hTERT mRNA. It is a powerful, accurate, specific, and sensitive method to measure the level of hTERT transcript $(19,20)$. In vitro, the minimum requirements for active telomerase are two components, hTER and hTERT, the presence of telomerase inhibitors such as proteases or RNases in tissue extract may affect the outcome sensitivity of the TRAP assay (20). Also evaluation of the copy numbers of hTERT mRNA by real time PCR makes statistical analysis more appropriate.

Early reports have detected telomerase activity, measured by TRAP assay, in colorectal carcinomas but not in normal mucosa $(13,14,21)$. Recently, telomerase activity or hTERT expression has been demonstrated in normal mucosa $(16,17,20)$. hTERT was also found to be expressed in non-tumour colorectal tissues by immunohistochemistry (24). A quantitative study found expression level of hTERT mRNA was higher in adjacent non-tumour than in tumour tissues, and 21\% of 57 tumour samples were expressed higher hTERT mRNA than in nontumour samples (25). In concurrence with the recent literature, we showed that telomerase activity, as expressed by hTERT copy number, was noted in normal tissue, adenoma and adenocarcinoma from the colon and the rectum $(16,17,20,25)$. More than half of the adenocarcinomas expressed higher level of hTERT than the morphological normal mucosa. The level of expression of hTERT in the colorectal adenocarcinomas was also slightly higher than in the adenomas though the difference did not reach statistical significant level.

The presence of hTERT mRNA in adjacent non-tumour colorectal mucosa could be explained by different hypothesis. Alternative splicing of hTERT transcript has been reported to 
regulate telomerase activity. Therefore, expression of hTERT transcript may not reflect the actual level of telomerase in non-tumour tissues (26). Infiltrating activated lymphocytes or cancer cells may also affect the outcome of hTERT. As telomerase is a common feature of abnormal epithelial cell proliferation during cancer development, expression of hTERT mRNA in non-tumour tissues may not be sufficient to activate telomerase (26). In this study, the presence of telomerase hTERT in dysplastic mucosa (adenoma) suggests that telomerase is activated early in the carcinogenesis. Thus, in morphological non-tumour colorectal mucosa, the presence of low level of telomerase hTERT may indicate that carcinogenesis start in this mucosa before any detectable morphological changes noted by pathologists.

In the literature, conflicting results were reported for correlation of telomerase or hTERT expression with clinical and pathological parameters $(15,18,22)$. Some studies carried out in colorectal cancer have correlated presence of telomerase expression either with depth of tumour (pT) $(16,22)$, tumour grade $(17,25)$, or with stage (18). Other studies found no correlation between telomerase expression and prognosis despite steady increased in telomerase expression during cancer development $(13,15,27)$. In this study, hTERT level in colorectal adenocarcinomas was not related to the age and gender of the patients. The quantitative results revealed that the hTERT mRNA level was increased in tumour carcinomas during cancer progression from normal morphology to dysplastic tissue (adenoma) to cancer (adenocarcinoma). However, our data did not confirm the statistical difference between hTERT mRNA expression and tumour status, lymph node, stage and grade. In tumour with distant metastases, the level of telomerase hTERT expression was significantly increased in tumour tissue samples when compared with normal, but no statistical correlation was found. This result should be interpreted with caution due to small number of samples with distant metastases $(n=3)$. 
In the recent years, researchers have demonstrated that cancers located in the right (proximal) and left (distal) colorectum can be distinguished by clinical and molecular criteria [28-30]. Proximal colorectal tumours, when compared to distal tumours, were more often found in older age, females and benefit from 5-fluorouracil-based- chemotherapy. Right sided tumours also showed significantly less nuclear $\beta$-catenin and p53 overexpression than left-sided tumours [28-30]. In this study, a significant difference was found between hTERT expression and the location of the tumour ( $\mathrm{p}=0.029$ ), which indicates that proximal tumours in the colorectum are more likely to be low hTERT expression than the distal tumours. This is the first study reporting statistical significance between hTERT mRNA expression and the 2 clinical locations of the tumour. The difference may be related to difference of carcinogenesis from the 2 clinical locations which can also be deduced from their known difference in the clinical behaviour.

To conclude, colorectal adenocarcinomas in North Queensland revealed expression of telomerase hTERT mRNA, which was detected quantitatively by real-time PCR. The expression level in colorectal adenocarcinoma was different from non-tumorous mucosa and with the location of the cancer. The latter could account for their known difference in the clinical behaviour of the carcinomas emerged from different sites in the colon \& rectum. hTERT might be a potential biomarker for colorectal adenocarcinoma. 


\section{References}

1. Vogelstein B, Kinzler KW. The multistep nature of cancer. Trends Genet. 1993; 9:138-41.

2. Vogt PK. Cancer genes. West J Med. 1993;158: 273-78.

3. Blackburn EH. Telomerases. Annu Rev Biochem 1992; 61:113-129.

4. Nugent CI, Lundblad V. The telomerase reverse transcriptase: components and regulation. Genes Dev 1998; 12: 1073-1085.

5. Shay JW, Wright WE. The reactivation of telomerase activity in cancer progression. Trends Genet 1996;12:129-131.

6. Holt SE, Shay JE. Role of telomerase in cellular proliferation and cancer. J Cell Physio. 1999; 180: 10-18.

7. Wright WE, Shay JW. Cellular senescence as a tumor-protection mechanism: the essential role of counting. Curr Opin Genet Dev 2001; 11: 98-103.

8. Kim NW, Piatyszek MA, Prowse KR, et al. Specific association of human telomerase activity with immortal cells and cancer. Science 1994; 266: 2011-15.

9. Shay JW, Bacchetti S. A survey of telomerase activity in human cancer. Eur J Cancer 1997; 33: 787-91.

10. Feng J, Funk WD, Wang SS, et al. The RNA component of human telomerase. Science 1995; 269: 1236-41.

11. Meyerson M, Counter CM, Eatom EN, et al. hEST2, the putative human telomerase catalytic subunit gene, is up-regulated in tumor cells and during immortalization. Cell 1997; 90: 785-95.

12. Nakayama J, Tahara H, Tahara E, et al. Telomerase activation by hTRT in human normal fibroblasts and hepatocellular carcinoma. Nat Genet 1998; 18: 65-68.

13. Fang DC, Joanne Y, Luo Y, et al. Detection of telomerase activity in biopsy samples of colorectal cancer. J Gastroenterol Hepatol 1999;14:328-332.

14. Yan P, Saraga EP, Bouzourene H, et al. Telomerase activation in colorectal cacinogenesis. J Pathol 1999; 189: 207-12.

15. Niiyama H, Mizumoto K, Sato N., et al. Quantitative analysis of hTERT mRNA expression in colorectal cancer. Am J Gastroenterol 2001; 96: 1895-1900. 
16. Shoji Y, Yoshinaga K, Inoue A, Iwasaki A, et al. Quantification of telomerase activity in sporadic colorectal carcinoma. Cancer 2000; 88: 1304-9.

17. Kanamaru T, Tanaka KI, Kotani J, et al. Telomerase activity and hTERT in development and progression of adenoma to colorectal cancer. Int J Mol Med 2002;10: 205-10.

18. Ghori A, Usselmann B, Ferryman S, et al. Telomerase expression of malignant epithelial cells correlates with Dukes’ stage in colorectal cancer. Colorectal Dis. 2002; 4: 441-46.

19. Bustin SA. Absolute quantification of mRNA using real-time reverse transcription polymerase chain reaction assays. J Mol Endocrinol. 2000; 25: 169-193.

20. De Kok JB, Ruers TJ, van Muijen GN, et al. Real-Time quantification of human telomerase reverse transcriptase mRNA in tumors and healthy tissues. Clin Chem 2000; 46: 313-18.

21. Cheng AJ, Tang RP, Wang JY, et al. Possible role of telomerase activation in the cancer predisposition of patients with hereditary nonpolyposis colorectal cancers. $J$ Natl Cancer Instit 1998; 90: 316-21.

22. Kawanishi-Tabata R, Lopez, F, Fratantonio S, et al. Telomerase activity in stage II colorectal carcinoma. Cancer 2002; 95: 1834-39.

23. Myung SJ, Yang SK, Chang HS, et al. Clinical usefulness of telomerase for the detection of colon cancer in ulcerative colitis patients. J Gastroenterol 2005; 20: 1578-83.

24. Tahara H, Yasui W, Tahara E, et al. Immuno-histochemical detection of human telomerase catalytic component, hTERT, in colorectal tumor and non-tumor tissue sections. Oncogene 1999;18: 1561-67.

25. Gertler R, Rosenberg R, Stricker D, et al. Prognostic potential of the telomerase subunit human telomerase reverse transcriptase in tumor tissue and nontumorous mucosa from patients with colorectal carcinoma. Cancer 2002; 95: 2103-2111.

26. Ulaner GA, Hu JF, Vu TH, et al. Regulation of telomerase by alternative splicing of human telomerase reverse transcriptase (hTERT) in normal and neoplastic ovary endometrium and myometrium. Int J Cancer 2000; 85: 330-35. 
27. Lledo, SM, Garcia-Granero E, Dasi F, et al. Real time quantification in plasma of human telomerase reverse transcriptase (hTERT) mRNA in patients with colorectal cancer. Colorectal Dis 2004; 6 :236-42.

28. Iacopetta B. Are there two sides to colorectal cancer? Int J Cancer 2002;101:403-8.

29. Kapiteijn E, Liefers GJ, Los LC, et al. Mechanisms of oncogenesis in colon versus rectal cancer. J Pathol 2001;195:171-78.

30. Birkenkamp-Demtroder K, Olesen SH, et al. Differential gene expression in colon cancer of the caecum versus the sigmoid and rectosigmoid. Gut. 2005;54:374-84. 


\section{Figures legends}

Figure 1. A. Quantitation data of rotor gene for tumour and adjacent mucosa samples from patients with colorectal cancer. B. Standard curve was constructed from 6 known concentration of hTERT segment (copy numbers range $1.2 \times 10^{1}-1.2 \times 10^{6}$ ).

Figure 2. Expression level of hTERT mRNA in tumour tissues and adjacent normal mucosa. In the box, horizontal lines represent the median value and the edges represent $25^{\text {th }}$ and $75^{\text {th }}$ percentiles, respectively. The whiskers indicate the minimum and maximum value. 
Figure 1

A

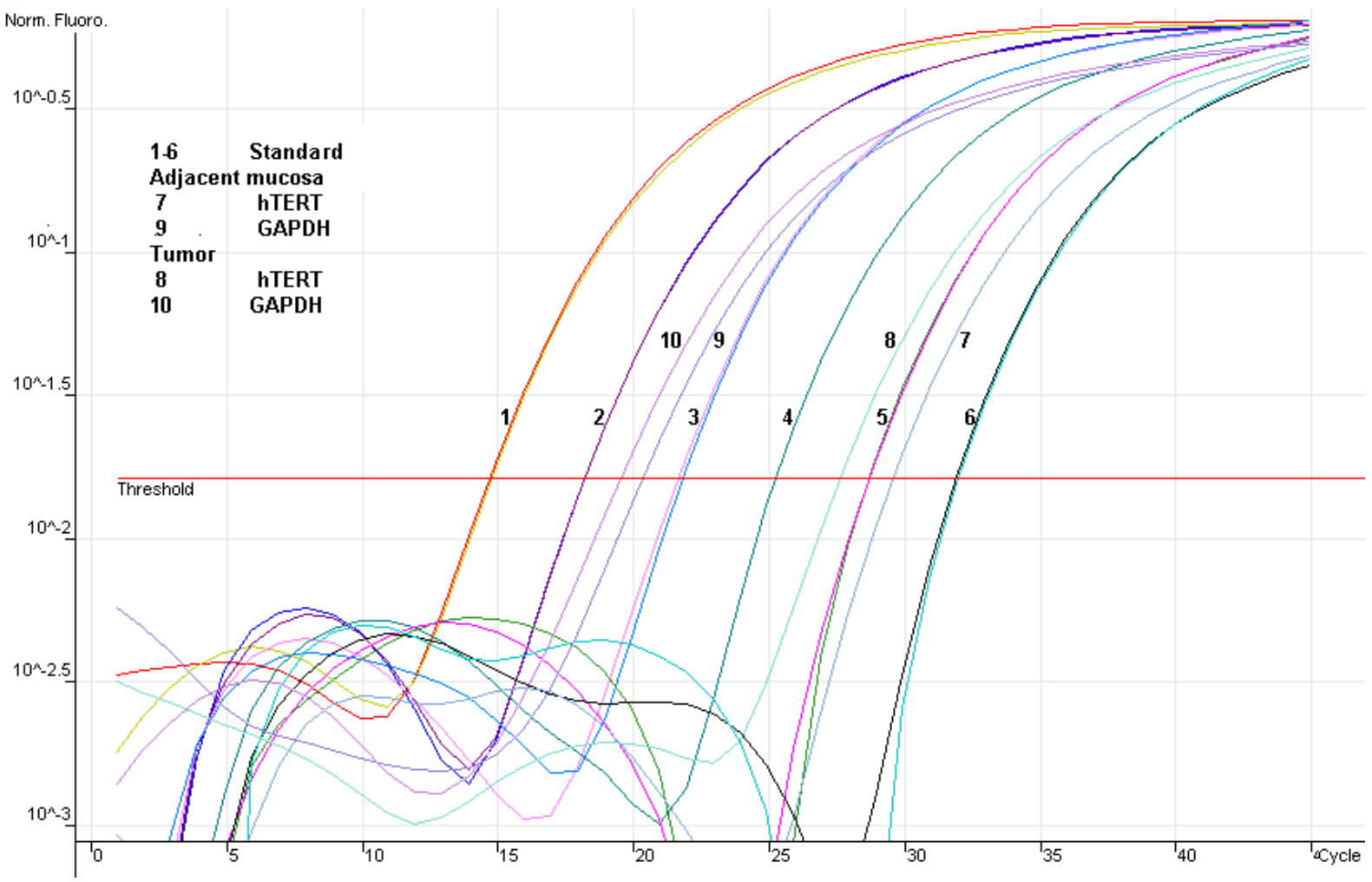

B

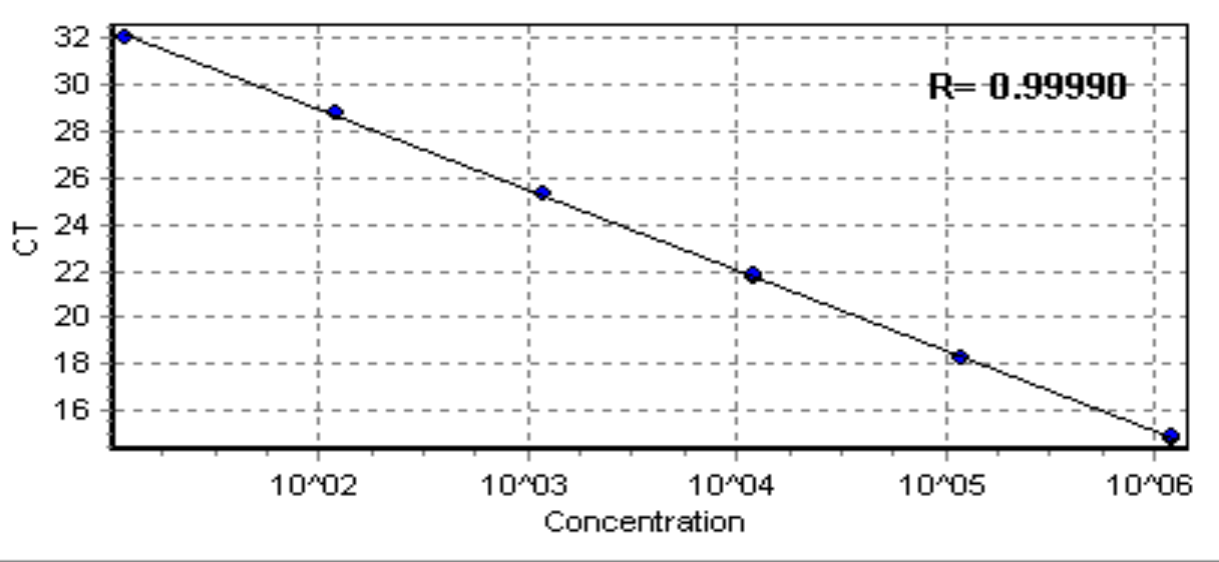


Figure 2

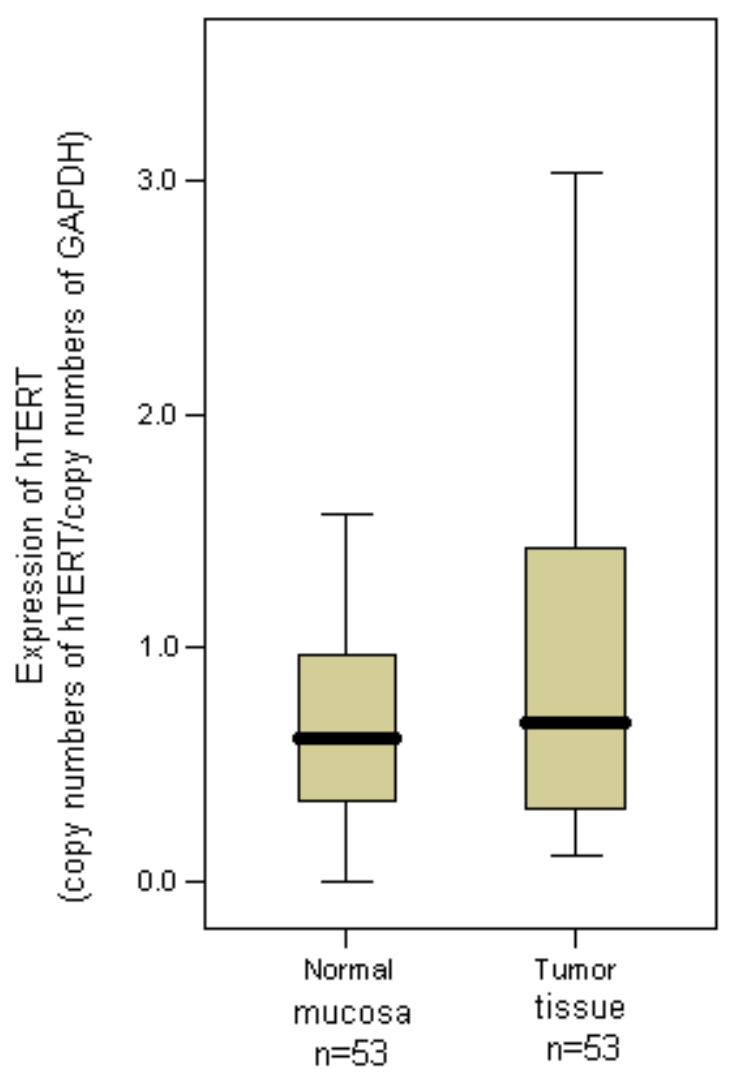


Table 1. PCR primers and probes used for quantitation of $h T E R T$ and GAPDH.

\begin{tabular}{|lcc|}
\hline Primer & Sequence & Amplicon size, bp \\
\hline hTERT & & \\
Upper & 5'-GCATTGGAATCAGACAGCAC-3' & \\
Lower & 5'-CCACGACGTAGTCCATGTTC-3' & 165 \\
Probe & 5'-(FAM) CGCCCTGCTGACGTCCAGAC (TAMRA)-3' & \\
GAPDH & & \\
Upper & 5'-ATGGGTGTGAACCATGAGAA-3' & \\
Lower & 5'-GTGCTAAGCAGTTGGTGTG-3' \\
Probe & 5'-(FAM) CCTCAAGATCATCAGCAATGCCTCC (TAMRA)-3' & \\
& & \\
& & \\
\hline
\end{tabular}


Table 2. Correlation between hTERT mRNA expression level and clinicopathological parameters for the patients with colorectal cancer

\begin{tabular}{|c|c|c|c|c|c|c|c|}
\hline \multirow[b]{3}{*}{ Variable } & \multicolumn{7}{|c|}{ hTERT } \\
\hline & \multicolumn{3}{|c|}{ Mucosa } & \multirow[b]{2}{*}{$P$} & \multicolumn{3}{|c|}{ Tumor } \\
\hline & No (\%) & Median & Range & & Median & Range & $P$ \\
\hline \multicolumn{8}{|l|}{ Gender } \\
\hline Male & $32(60)$ & 0.637 & $0.00-1.552$ & & 0.639 & $0.111-2.919$ & \\
\hline Female & 21 (40) & 0.577 & $0.00-1.584$ & n.s & 0.852 & $0.150-3.033$ & n.s \\
\hline \multicolumn{8}{|l|}{ Age } \\
\hline$<65$ & $22(42)$ & 0.715 & $0.00-1.584$ & & 0.798 & $0.171-2.919$ & \\
\hline$\geq 65$ & 31 (58) & 0.577 & $0.00-1.492$ & n.s & 0.603 & $0.111-3.033$ & n.s \\
\hline \multicolumn{8}{|l|}{ Site } \\
\hline Colon & $36(68)$ & 0.637 & $0.00-1.584$ & & 0.841 & $0.111-3.033$ & \\
\hline Rectum & $17(32)$ & 0.577 & $0.00-1.552$ & n.s & 0.664 & $0.150-2.073$ & n.s \\
\hline \multicolumn{8}{|l|}{ Tumor size } \\
\hline$<4 \mathrm{~cm}$ & $21(40)$ & 0.716 & $0.00-1.584$ & & 0.852 & $0.150-2.622$ & \\
\hline$\geq 4 \mathrm{~cm}$ & $32(60)$ & 0.519 & $0.00-1.552$ & n.s & 0.438 & $0.111-3.033$ & n.s \\
\hline \multicolumn{8}{|c|}{ Tumor status } \\
\hline $\mathrm{T} 1$ & $7(13)$ & 0.544 & $0.227-0.972$ & & 1.154 & $0.233-1.905$ & \\
\hline $\mathrm{T} 2$ & $12(23)$ & 0.807 & $0.00-1.584$ & & 0.677 & $0.200-2.622$ & \\
\hline T3 & $31(58)$ & 0.562 & $0.00-1.201$ & & 0.675 & $0.111-3.033$ & \\
\hline $\mathrm{T} 4$ & $3(6)$ & 0.942 & $0.380-0.985$ & n.s & 1.415 & $0.462-1.911$ & n.s \\
\hline \multicolumn{8}{|c|}{ Lymph node status } \\
\hline NO & $38(72)$ & 0.670 & $0.00-1.584$ & & 0.742 & $0.150-3.033$ & \\
\hline $\mathrm{N} 1$ & $15(28)$ & 0.380 & $0.00-1.552$ & n.s & 0.462 & $0.111-2.483$ & n.s \\
\hline \multicolumn{8}{|l|}{ Stage } \\
\hline 1 & $16(30)$ & 0.761 & $0.227-1.584$ & & 1.105 & $0.200-2.622$ & \\
\hline II & $20(38)$ & 0.596 & $0.00-1.175$ & & 0.508 & $0.150-3.033$ & \\
\hline III & $14(26)$ & 0.408 & $0.00-1.552$ & & 0.557 & $0.111-2.483$ & \\
\hline IV & $3(6)$ & 0.714 & $0.380-0.942$ & n.s & 1.415 & $0.462-1.570$ & n.s \\
\hline \multicolumn{8}{|l|}{ Grade } \\
\hline 1 & $6(11)$ & 0.601 & $0.238-1.492$ & & 0.650 & $0.233-2.313$ & \\
\hline 2 & $39(74)$ & 0.577 & $0.00-1.584$ & & 0.685 & $0.111-3.033$ & \\
\hline 3 & $8(15)$ & 0.779 & $0.191-1.552$ & n.s & 0.774 & $0.312-2.919$ & n.s \\
\hline \multicolumn{8}{|l|}{ Metastasis } \\
\hline MO & $50(94)$ & 0.596 & $0.00-1.584$ & & 0.680 & $0.111-3.033$ & \\
\hline M1 & $3(6)$ & 0.714 & $0.380-0.942$ & n.s & 1.415 & $0.462-1.570$ & n.s \\
\hline
\end{tabular}


Table 3. Relationship between hTERT and the location of the adenocarcinoma

\begin{tabular}{llll}
\hline \multicolumn{4}{c}{ Tumor colorectal } \\
\hline & $\begin{array}{l}\text { Proximal } \\
\text { No (\%) }\end{array}$ & $\begin{array}{l}\text { Distal } \\
\text { No (\%) }\end{array}$ & P value \\
\hline hTERT $_{\text {copy number }}$ & & & \\
$<1$ & $12(23 \%)$ & $20(38 \%)$ & \\
$\geq 1$ & $2(4 \%)$ & $19(36 \%)$ & 0.029 \\
\hline
\end{tabular}

Proximal: caecum, ascending colon and transverse colon

Distal: descending colon, sigmoid colon and rectum 